\title{
EEG Epilepsy Seizure Signal Characterization through theories of Random Matrix Analysis
}

\author{
Mayukha $\mathrm{Pal}^{1}$, and P. Madhusudana Rao ${ }^{2}$
}

\begin{abstract}
We investigated the fractal properties of EEG time series signals of healthy \& epilepsy patients for characterization using the correlation matrix from Random Matrix theory. It is observed from our result that the time series of EEG signals from the subject of study could be used in real time application to characterize time series signals of an epilepsy patient with seizure from a healthy subject or patient who does not contain seizure using our analysis methodology.
\end{abstract}

Keywords - EEG time series, Epilepsy, Random Matrix Theory, Correlation Matrix, Eigen Value.

\section{INTRODUCTION}

$\mathrm{D}$ YNAMICAL systems found in nature are complex and show presence of chaotic behavior, nonlinearity, and exhibit long-range correlations [1-3]. Significance of quantifying the long-range correlation of such systems is to have deep understanding of the dynamics of the parameters underlying the complex system which could be studied using methods like fractal analysis [4]. Human brain has the ability to perform very sophisticated cognitive tasks hence analysis of signals from neuronal network of human brain whose structure is highly complicated often referred as complex system can be studied using suitable fractal study as it has ability to characterize systems that are basically irregular at all scales. In recent past large amount of scientific study on brain dynamics was evolved using the signals measured from brain which is generated due to cooperative interactions among widely distributed, densely interconnected active neurons. It has become common to apply principles derived from nonlinear dynamics and statistical mechanics to characterize these neuronal systems [5-10]. Correlation study using fractal geometry on electroencephalogram (EEG) signals reveals that the underlying pattern of neuronal activation in term of EEG signals are non-stationary with self-affine dynamics and nonlinear characteristics [11-14]. Many studies were known to be reported using different methods like permutation entropy, support vector machine, granger causality from the literature which were applied to different kinds of EEG data from human to characterize brain signals of different pathological states [15-19].

Corresponding author: Mayukha Pal is with India Innovation Center, General Electric Company, Secunderabad-500003, India

P. Madhusudana Rao is with JNTUH College of Engineering, Jawaharlal Nehru Technological University, Hyderabad-500085, India.
It is witnessed that random matrix theory approach for statistical analysis of cross-correlation matrix has brought attention of researcher to analyze natural time series data especially from financial and biological systems [20-26]. The purpose of this paper is to propose a real time analysis technique to characterize epilepsy patients with seizure from healthy human or patients who achieved complete seizure control using EEG signals that are measured for different recordings simultaneously making a high dimensional data for analysis using the random matrix theory. The chapter 2 of the paper describes the data and methodology while chapter 3 discusses about results and discussion of our work.

\section{Data Analysis Methodology}

We have obtained the EEG data of healthy \& epilepsy seizure patient from the Dept. of Clinical Epileptology, University Hospital of Bonn, Germany (http://www.meb.unibonn.de/epileptologie/science/physik/eeg data.html) [10]. Data consists of five sets (denoted A-E) each containing 100 single channel EEG segments having N=4096 samples points. The description of EEG data sets under study is given below:

Set A: Using standardized electrode placement scheme extra-cranial recording of healthy volunteers with eyes open

Set B: Using standardized electrode placement scheme extra-cranial recording of healthy volunteers with eyes closed

Set C: Intracranial recordings from the hippocampal formation of the opposite hemisphere of the brain of patients during seizure free interval

Set D: Intracranial recordings from within epileptogenic zone of patients during seizure free interval

Set E: Recordings from patients contained seizure activity 


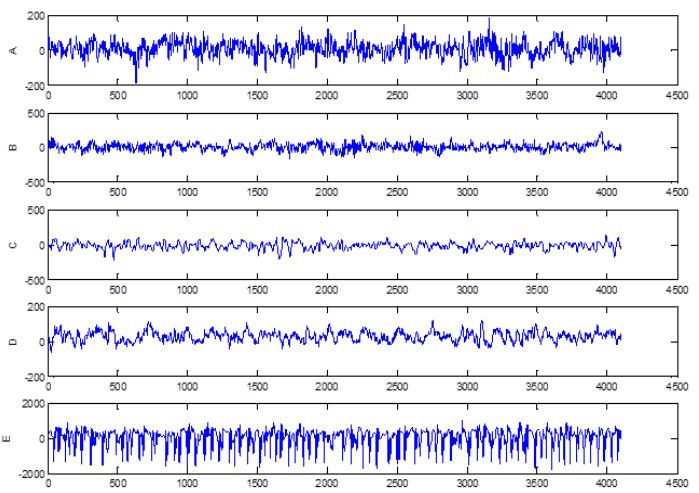

Fig. 1 EEG time series data from each of the five sets. Amplitudes of surface EEG recordings are typically in the order of some $\mathrm{mV}$

From the mentioned data collection procedure [10], each of the five sets (A-E) contains 100 single channel EEG segments of 4096 sample points each. For our analysis of characterizing healthy subject with patients suffering from epilepsy we choose data set A, B and E for our future study. Since the selected patients belonged to different socioeconomic and age groups we have carried out the mean subtraction to remove the average part of the signal and normalized it by diving over standard deviation. This formed our initial 100 x 4096 data matrix for correlation matrix study. We had taken those obtained matrixes of each data set A, B and E to study the multifractal properties for EEG signal characterization using Random matrix theory (RMT) [20-24] for analysis purpose which could be described as below:

Now we wish to apply the theory of random matrix which many literatures unveil its potentials to understand the statistical structure of the empirical correlation matrices that are appearing in the study of multivariate time series analysis [20-23]. Here now we take the initially obtained 3 sets of 100 x 4096 data matrix in order to process for our analysis. Then the data is normalized to have unit variance as we had obtained the mean value of the data for each row of the matrix from each data set by taking the average. This was then subtracted from each data set to get $\delta \mathrm{x}_{\mathrm{i}}(\mathrm{t})$. Here i represents the no. of channels while $t$ the sample number. We have computed the standard deviation which was then divided with the mean subtracted data to obtain the correlation matrix for our correlation study.

$$
\mathrm{C}_{\mathrm{ij}}=\frac{1}{\mathrm{~T}} \sum_{\mathrm{t}=1}^{\mathrm{T}} \delta \mathrm{x}_{\mathrm{i}}(\mathrm{t}) \delta \mathrm{x}_{\mathrm{j}}(\mathrm{t})
$$

where $A_{i t}^{T}=\delta x_{i}(t), M$ is a $N \times T$ rectangular matrix and ${ }^{T}$ denotes matrix transposition then the correlation matrix is $\mathrm{C}=$ $\left(M^{T} M\right) / T$. The $p c(\lambda)$ density of eigen values of $C$ is defined as

$$
\mathrm{pc}(\lambda)=\frac{1}{\mathrm{~N}} \frac{\mathrm{dn}(\lambda)}{\mathrm{d} \lambda}
$$

where $n(\lambda)$ is the number of eigen values of $C$ less than $\lambda$. If $M$ is random matrix of $T \times N$ then $\rho c(\lambda)$ is self-averaging where $\mathrm{N} \rightarrow \infty, \mathrm{T} \rightarrow \infty$, and $\mathrm{Q}=\mathrm{T} / \mathrm{N} \geq 1$.

$$
\text { Hence, } p c(\lambda)=\frac{Q}{2 \pi \sigma^{2}} \frac{\sqrt{\left(\lambda_{\max }-\lambda\right)\left(\lambda-\lambda_{\min }\right)}}{\lambda}
$$

where $\lambda_{\min }^{\operatorname{mgx}}=\sigma^{2}\left(1+\frac{1}{Q} \pm 2 \sqrt{Q}\right), \lambda$ varies between $\lambda_{\min }$ and $\lambda_{\max }$, and $\sigma^{2}$ is the variance of elements of $\mathrm{M}$ and is unity with the normalization. Then we had taken these obtained correlation matrix of each data set A, B and E to study the statistical properties for EEG signal characterization. Upon diagonalizing the matrix $\mathrm{C}$ i.e. $\mathrm{Cu}_{\mathrm{k}}=\lambda_{\mathrm{k}} \mathrm{u}_{\mathrm{k}}$ we could get the corresponding eigenvalues $\lambda_{\mathrm{k}}(\mathrm{k}=1,2, \ldots, \mathrm{N})$ and its corresponding eigenvectors $u_{k}$. Then the Eigen values of $C$ are rank-ordered from the smallest to the largest for our further analysis purpose. In random matrices, one observes the universal functional forms for eigenvalue correlations that depend only on the general symmetries of the matrix. The universal properties of the data could be tested by finding a transformation called "unfolding" which maps the eigenvalues $\lambda_{k}$ to new variables called "unfolded eigenvalues" $\xi_{\mathrm{k}}$ having uniform distribution. These unfolding ensures that the distances between eigenvalues are expressed in units of local mean eigenvalue spacing hence allows comparison with analytical results.

To calculate the unfolding let us first define the cumulative distribution function of eigenvalues which counts the number of eigenvalues in the interval $\lambda_{\mathrm{k}} \leq \lambda$;

$$
F(\lambda)=N \int_{-s e}^{\lambda} p(\lambda) d \lambda
$$

where $\rho(\lambda)$ is the probability density of eigen values and $N$ is the total number of eigenvalues. The function $F(\lambda)$ can be decomposed into an average and a fluctuating part as below,

$$
F(\lambda)=F_{a v}(\lambda)+F_{\text {fluc }}(\lambda)
$$

Since $\frac{d F_{\text {fluc }}(\lambda)}{d \lambda}=0=\rho_{\text {fluc }}$ hence the dimensionless unfolded eigenvalues are then given by $\xi_{\mathrm{k}}=\mathrm{F}_{\mathrm{av}}\left(\lambda_{\mathrm{k}}\right)$. Now to find $\mathrm{F}_{\mathrm{av}}(\lambda)$ one fit the cumulative distribution function $F(\lambda)$ with the analytical expression for $\rho_{c}(\lambda)$ for obtaining the unfolded eigenvalues.

\section{RESUltS AND DisCUSSION}

The theory of random matrix (RMT) explained in our method applied to analyze the data which has shown characterizing the EEG signals of healthy from the patients with epilepsy seizure. We had plotted the nearest-neighbor spacing distribution $\mathrm{P}(\mathrm{s})$ of the unfolded eigen values $\xi_{\mathrm{k}}$. We find a very good agreement in the fitting for all data sets except for the data $\mathrm{E}$ where we have observed two peaks whereas the healthy subjects A and B contains single peak in the wigner distribution. Similarly the highest eigen vector was plotted for all 3 data sets and we could clearly observe from figure 2 the existence of two peaks in the case of signal with seizure activity from epilepsy patients while we observe a single peak from healthy subjects. 



(A)
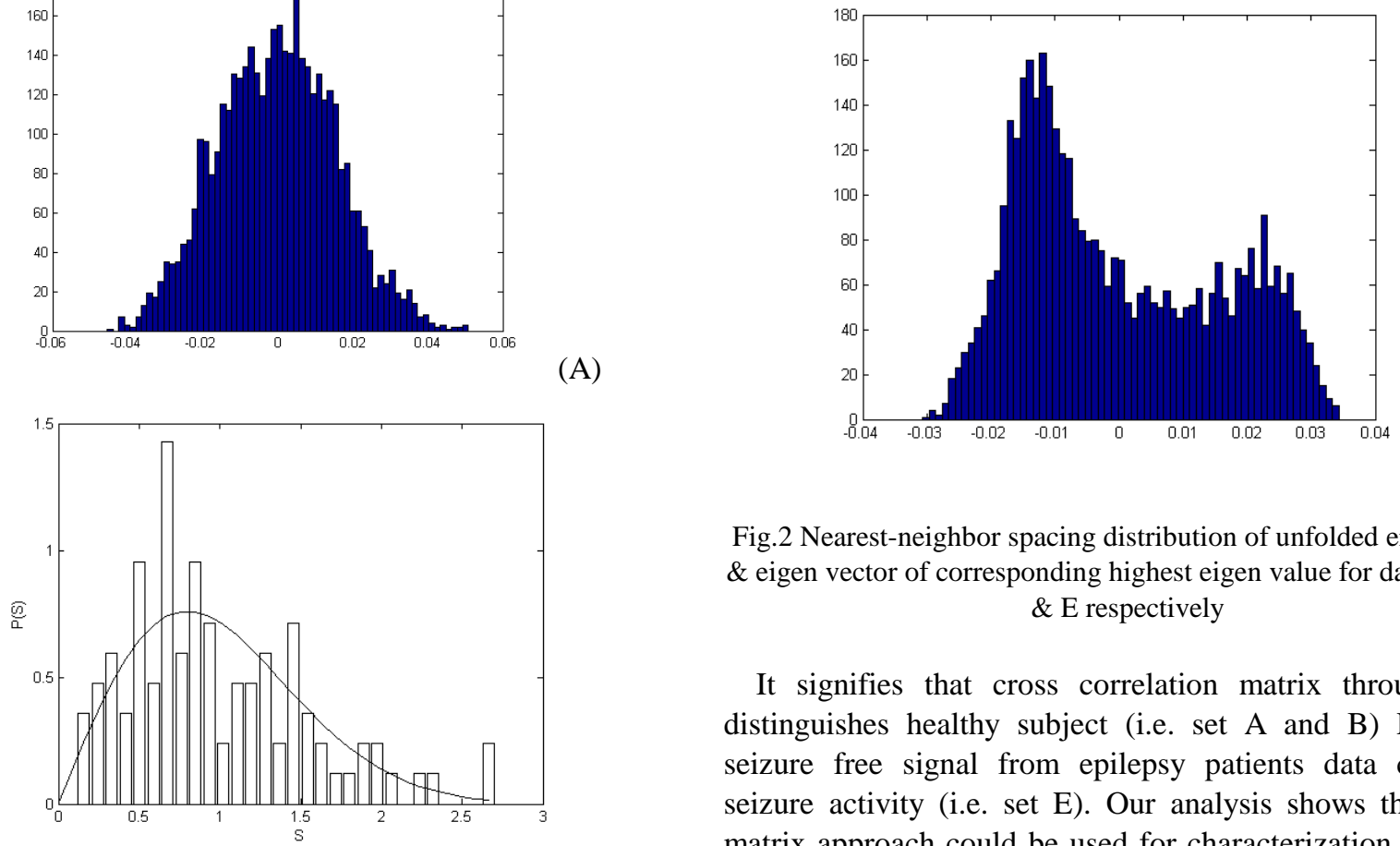

(E)

Fig.2 Nearest-neighbor spacing distribution of unfolded eigenvalues \& eigen vector of corresponding highest eigen value for data set A, B $\&$ E respectively

It signifies that cross correlation matrix through RMT distinguishes healthy subject (i.e. set $\mathrm{A}$ and B) EEG with seizure free signal from epilepsy patients data containing seizure activity (i.e. set E). Our analysis shows the random matrix approach could be used for characterization, diagnosis and prognosis of patients with epilepsy from the EEG signals.

\section{ACKNOWLEDGMENTS}

The author MP would like to thank C R Rao AIMSCS, Hyderabad for providing the research facility to carry out this work.

\section{REFERENCES}

[1] H.E. Hurst, A suggested statistical model of some time series which occur in nature, Nature 180, 494 (1957). http://dx.doi.org/10.1038/180494a0

[2] B.B. Mandelbrot, The Fractal Geometry of Nature, W.H. Freeman, New York, (1982).

(B)

[3] A.L. Barabasi, and T. Vicsek, Multifractality of self-affine fractals, Phys Rev A 44, pp. 27302733 (1991). http://dx.doi.org/10.1103/PhysRevA.44.2730

[4] A. Bunde, and S. Havlin, Fractals in Science, Springer-Verlag, Heidelberg, Germany (1995).

[5] J. Gao, J. Hu, and W-w Tung, Complexity measures of brain wave dynamics. Cognitive Neurodynamics 5: 171-182 (2011). http://dx.doi.org/10.1007/s11571-011-9151-3

[6] C.J. Stam, Nonlinear dynamical analysis of EEG and MEG: Review of an emerging field. Clinical Neurophysiology 116: 2266-2301 (2005). http://dx.doi.org/10.1016/j.clinph.2005.06.011 
[7] A.-M Lazar, and R. Ursulean, Further Applications of the Fractal Spectra of the EEG Signals, Electronics and Electrical Engineering, No. 2(82) ISSN 1392-1215 (2008).

[8] P.L. Nunez, Brain, mind, and the structure of reality, Oxford University Press: New York, 306 p (2010). http://dx.doi.org/10.1093/acprof:oso/9780195340716.001.0001

[9] Ayay Kaplan, A.A. Fingelkurts, A.A. Fingelkurts, S.V. Borisov, and B.S. Darkhovsky, Nonstationary nature of the brain activity as revealed by EEG/MEG: Methodological, practical and conceptual challenges. Signal Processing 85: 2190-2212 (2005). http://dx.doi.org/10.1016/j.sigpro.2005.07.010

[10] Ralph G. Andrzejak, Klaus Lehnertz, Florian Mormann, Christoph Rieke, Peter David, and Christian E. Elger, Indications of nonlinear deterministic and finite-dimensional structures in time series of brain electrical activity: Dependence on recording region and brain state, Physical Review E 64, 061907 (2001). http://dx.doi.org/10.1103/PhysRevE.64.061907

[11] P.L. Nunez, and R. Srinivasan, Electric Fields of the Brain: The Neurophysics of EEG, Oxford University Press: New York (2006). http://dx.doi.org/10.1093/acprof:oso/9780195050387.001.0001

[12] G. Solovey, K.J. Miller, J.G. Ojemann, M.O. Magnasco, and G.A. Cecchi, Self-Regulated Dynamical Criticality in Human ECoG. Front Integr Neurosci 6: 44 (2012). http://dx.doi.org/10.3389/fnint.2012.00044

[13] C.J. Stam, E.A. de Bruin, Scale-free dynamics of global functional connectivity in the human brain. Human Brain Mapping 22: 97-109 (2004). http://dx.doi.org/10.1002/hbm.20016

[14] J.-S. Lee, D. Spiegel, S.-B. Kim, J.-H. Lee, S.-I. Kim, Fractal Analysis of EEG in Hypnosis and its Relationship with Hypnotizability. International Journal of Clinical and Experimental Hypnosis 55: 14-31 (2007). http://dx.doi.org/10.1080/00207140600995810

[15] Li Jing, Yan Jiaqing, Liu Xianzeng, and Ouyang Gaoxiang, Using Permutation Entropy to Measure the Changes in EEG Signals During Absence Seizures, Entropy, 16, 3049-3061 (2014). http://dx.doi.org/10.3390/e16063049

[16] Abdulhamit Subasi, and M. Ismail Gursoy, EEG signal classification using PCA, ICA, LDA and support vector machines, Export Systems with Applications 37, 8659-8666 (2010). http://dx.doi.org/10.1016/j.eswa.2010.06.065

[17] N. Kannathal, U. Rajendra Acharya, C.M. Lim, P.K. Sadasivan, Characterization of EEG - A comparative study, Compuer Methods and Programs in Biomedcine 80, 17-23 (2005). http://dx.doi.org/10.1016/j.cmpb.2005.06.005

[18] Nicoletta Nicolaou, Saverios Hourris, Pandelitsa Alexandrou, Julius Georgiou, EEG-Based Automatic Classification of 'Awake' versus 'Anesthetized' state in General Anesthesia Using Granger Causality, PLoS one 7, 3, e33869 (2012)

[19] J.W. Kantelhardt, F. Gans, A.Y. Schumann and T. Penzel, EEG CrossModulation During Sleep and Wake, Proceedings of Biosignal Berlin, Germany (2010).

[20] A.M. Sengupta, P.P. Mitra, Distributions of singular values for some random matrices, Physical Review E 60, 3, 3389-3392 (1999). http://dx.doi.org/10.1103/PhysRevE.60.3389

[21] V. Plerou, P. Gopikrishnan, B. Rosenow, L.A.N. Amaral, and H.E. Stanley, A random matrix theory approach to financial crosscorrelations, Physica A 287, 374-382 (2000) http://dx.doi.org/10.1016/S0378-4371(00)00376-9.

[22] Laurent Laloux, Pierre Cizeau, Jean-Philippe and Marc Potters, Noise Dressing of Financial Correlation Matrices, Physical Review Letters 83, 7, 1467-1470 (1999). http://dx.doi.org/10.1103/PhysRevLett.83.1467

[23] Vasiliki plerou, Parameswaran Gopikrishnan, Bernd Rosenow, Luis A. Nunes Amaral and H.E. Stanley, Universal and Nonuniversal Properties of Cross Correlations in Financial Time Series, Physical Review Letters 83, 7, 1471-1474 (1999) http://dx.doi.org/10.1103/PhysRevLett.83.1471.

[24] Anita H. Gharekhan, Siddharth Arora, K.B.K. Mayya, Prasanta K. Panigrahi, M.B. Sureshkumar, and Asima Pradhan, Characterizing breast cancer tissues through the spectral correlation properties of polarized fluorescence, Journal of Biomedical Optics, 13(5) 054063 (2008). http://dx.doi.org/10.1117/1.2997376

[25] Gang-Jin Wang, Chi Xie, Shou Chen, Jiao-Jiao Yang, and Ming-Yan Yang, Random matrix theory analysis of cross-correlations in the US stock market: Evidence from Pearson's correlation coefficient and detrended cross-correlation coefficient Physica A 392, 3715-3730 (2013). http://dx.doi.org/10.1016/j.physa.2013.04.027

[26] Vasiliki Plerou, Parameswaran Gopikrishnan, Bernd Rosenow, Luis A. Nunes Amaral, Thomas Guhr, and H. E. Stanley, Random matrix approach to cross correlations in financial data Physical Review E, 65, 066126 (2002).

http://dx.doi.org/10.1103/PhysRevE.65.066126

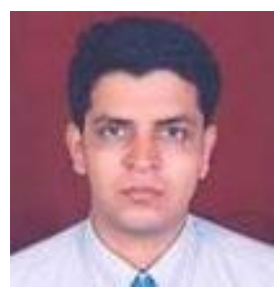

Mr. Mayukha Pal comes from Kandigaon, Odisha, India. He holds a master's degree from University of Hyderabad and is currently working as Engineering Technical Leader at India Innovation center of General Electric Company, Hyderabad. He is pursuing his Ph. D. at Jawaharlal Nehru Technological University in the area of Fluctuation Analysis, Nonlinear Time Series Analysis of Complex Systems, computational

Physics and data science

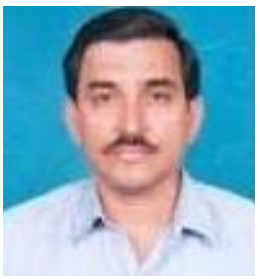

Prof. PolaMadhusudana Rao comes from Guntur, Andhra Pradesh, India. He holds a Ph.D. degree from University of Hyderabad and is currently working as Senior Professor at JNTUH College of Engineering, Jawaharlal Technological University, Hyderabad. His area of research interests are: Condensed Matter Physics, Nano composites, Conductive Polymers; Computational Physics Fluctuation Analysis, Nonlinear Time Series Analysis of Complex Systems, Multifractal Study. 